U. of Towa $68-15$

\title{
Response of a One-Dimensional Vlasov Plasma to External Electric Fields*
}

by

Rollin C. Harding

\author{
Department of Physics and Astronomy \\ The University of Iowa \\ Iowa City, Iowa
}

March 1968

*This work was supported in part by the National Aeronautics and space Administration under Grant No. NGR-16-001-043. 
ABSTRACT

The response of a one-dimensional electron plasma confined by perfectly reflecting boundaries has been calculated numerically for several externally-maintained electric field configurations representing different physical situations. The electron distribution function is expanded in a Fourier-Hermite series. The external fields (of various wavenumbers and frequencies) are represented by equivalent charge density terms in Poisson's equation.

In the first situation the external field is a standing wave oscillating at approximately the resonant frequency for the given wavenumber $k_{0}$. The energy of the particle distribution and the envelope of the particle electric field are found to oscillate slowly with a period corresponding to the period of oscillation of particles trapped in the potential trough of the driving wave. Analogous spatial oscillations have been reported by Malmberg and Wharton. The physical system represented in these calculations is similar to the experiment of Decker and Hirshfield in which a series of disks with alternating potential is set up perpendicular to the axis of a plasma column.

In the second situation the external source charge density is held constant and represents a small sinusoidal inhomogeneity in the ion background. The appropriate Bernstein, Greene, and Kruskal 
equilibrium is calculated and found numerically to be stable against small perturbations. Landau damping rates for perturbations from these inhomogeneous equilibria are compared with the values calculated by Jackson and Raether.

In another calculation the inhomogeneity in the background charge density is varied slowly compared with the electron plasma frequency to simulate the field seen by electrons in the presence of an ion acoustic wave. This is done to test the common assumption that in the presence of an ion wave the mobile electrons respond in such a way as to obey a locally isothermal equation of state. In the cases calculated the electrons do obey an isothermal equation of state to within a few percent.

In the fourth situation the ion inhomogeneity is switched on suddenly with the electrons initially in a uniform equilibrium. The electrons redistribute themselves to screen out the ion field and, if the inhomogeneity is not too large, they approach an inhomogeneous equilibrium by Landau damping. 


\section{INTRODUCTION}

We report here the results of numerical caloulations of the response of a collisionless electron plasma to an extennally-impcsed electric field which depends on space and time. Previous numerical. calculations by the same techniques ${ }^{1 \cdot 2 \cdot 3}$ have been concerned with the initial value problem for the same system. Inclusion of the external field allows statements to be made about the behavior of a Vlasov plasma in situations where the length and time scales of the phenomena bear no direct relation to the electron Debye length and electron plasma frequency; such situations often occur in practice.

The equations to be investigated are a generalization of the dimensionless equations treated by Armstrong: $1,2 \cdot 3$

$$
\begin{aligned}
& \frac{\partial f}{\partial t}+v \frac{\partial f^{\prime}}{\partial x}-E \frac{\partial f^{\prime}}{\partial v}=0, \\
& \frac{\partial E}{\partial x}=1-\int_{-\infty}^{\infty} f d v+\rho_{\text {ext. }}(x, t)
\end{aligned}
$$

where the electron Debye length is the fundamental unit of length and the reciprocal of the electron plasma frequency is the unit of 
time. $f=f(x, v, t)$ is the electron distribution, $E=E(x, t)$ is the electric field, and $\rho_{\text {ext. }}(x, t)$ is an external "source" charce density which is regarded as a given function of $x$ and $t$ and which is regarded as generating the "external" part of the total electric field. For example, $1+\rho_{\text {ext. }}$ might represent the charge density of the positive ions of a plasna; or pext. miknt stand for the quasi-electrostatic external disk structure in the experiment of Decker and Hirshfield. ${ }^{4}$ The spesific choice of the functional form is dictated by the problem one is attempting to simulate.

Calculations were done for four separate problems. After a brief summary of the method of computation in Section 2, we treat in Section 3 the response of an electron plasma to an external field which varies on the time scale of the electron plasma frequency. Section 4 describes the construction of inhomogeneous equilibria, and the study of Lardau damping of perturbations on these equilibria. Section 5 concerns the resporse of a plasma to a $\rho_{\text {ext. }}$ which varies slowly compared to the electron plasma frequency $\left(\omega_{0}<<\omega_{p}\right)$, with most of the attention directed to the degree to which the electron plasma obeys a locally isothermal equation of state in the presence of such a slowly varying external potential. Section 6 treats the time dependence of the shielding cloud which forms around an instantaneously introduced "test charge" distribution. 


\section{COMPUTATIONAI METHOD}

We shall choose initial conditions and functional forms for which the plasma may be alternatively regarded as spatially periodic or as obeying perfectly-reflectine boundary conditions at, $x=0$ and $x=L$. If we start with the electron distribution $f(x, v, 0)=f(-x,-v, 0)$ and external charge $\rho_{\text {ext. }}(x, t)=\rho_{\text {ext. }}(-x, t)$. with $p_{\text {ext, }}$ and $f(x, v, 0)$ being periodic with period $2 \mathrm{~L}$ in $\mathrm{x}$, then it is readily shown that $f(0, v, t)=f(0,-v, t)$ and $f(L, v, t)=$ $f(I,-v, t)$ for all $t>0$; the plasma is therefore specularly reflected at $x=0$ and $x=I$. It also follows that $E(O, t)=E(L, t)=0$ for all t.

The quantities $f, E$, and $\rho_{\text {ext. }}$ are all expanded in complex Fourier series,

$$
\begin{aligned}
& f(x, v, t)=\sum_{n=-\infty}^{\infty} f_{n}(v, t) e^{i n k_{0} x} . \\
& E(x, t)=\sum_{n=-\infty}^{\infty} E_{n}(t) e^{i n k_{0} x}, \\
& \rho_{\text {ext. }}(x, t)=\sum_{n=-\infty}^{\infty} \rho_{n}(t) e^{i n k_{0} x},
\end{aligned}
$$


where $k_{0}=\pi / L$. The symmetry conditions imply $f_{n}(-v, t)=f_{-n}(v, t)$, $E_{n}(t)=-E_{-n}(t)$, and $\rho_{n}(t)=\rho_{-n}(t)$, so we need compute only for $\mathrm{n} \geq 0$.

The $f_{n}$ are further expanded in the Gram-Charlier series in velocity used previously ${ }^{1,2,3}$ :

$$
f_{n}(v, t)=e^{-v^{2} / 2} \sum_{m=0}^{\infty} z_{m n}(t) h_{m}(v)
$$

where

$$
h_{m}(v)=\frac{(-1)^{m} e^{v^{2} / 2}}{\left(m !(2 \pi)^{\frac{1}{2}}\right)^{\frac{1}{2}}} \frac{d^{m}\left(e^{-v^{2} / 2}\right)}{d v^{m}}
$$

is the orthonormal Hermite polynomial of degree $\mathrm{m}$. In this representation a Maxwellian velocity distribution requires only the $\mathrm{m}=0$ term of the series.

$$
\begin{aligned}
& \text { Poisson's Equation (2) becones } \\
& E_{n}(t)=-\frac{(2 \pi)^{\frac{1}{4}} z_{o n}(t)}{i n k_{0}}+\frac{p_{n 1}(t)}{i n k_{o}},
\end{aligned}
$$

and substitution into the transformed version of Equation (1) gives 


$$
\begin{aligned}
\frac{d z_{m n}(t)}{d t} & =-i n k_{0}\left(\sqrt{m} z_{m-1, n}+\sqrt{m+1} z_{m+1, n}\right) \\
& -\sum_{\substack{q=-\infty \\
q \neq n}}^{\infty} \frac{i \sqrt{m}}{(n-q) k_{0}}\left\{(2 \pi)^{\frac{1}{4}} z_{0, n-q}-o_{n-q}\right\} z_{m-1, q},
\end{aligned}
$$$$
-\infty<n<\infty, m \geq 0,
$$

with $\mathrm{Z}_{-1, \mathrm{n}} \equiv 0$. The symmetry relations determine that

$$
z_{m,-n}(t)=z_{m, n}^{*}(t)=(-I)^{m} z_{m n}(t)
$$

The elements of the $\mathrm{Z}_{\mathrm{mn}}$ matrix are advanced step by step in time according to Equation (6), using Gill's specialization of the Runge-Kutta method.

Initial distributions are usually chosen to be Maxwellian in velocity so the only non-zero coefficients are $z_{\text {on }}$ Equation (6) guarantees, however, that elements with increasing $m$ will soon be non-zero. (In practice this is due mainly to the streaming term the first term on the right hand side of Equation (6)). As time increases it is necessary to calculate more and more terms in the Hermite series in order adequately to represent 
the velocity-space wrinkles in f. Typically, several hundred terms in the series are needed, as discussed previously by Armstrong. I, a In the Fourier expansion, disturbances spread to increasing $\mathrm{n}$ only slowly, and for the problems considered here the $\mathrm{n}=0,1$, and 2 values of the $z_{m n}$ are sufficient. 


\section{RESPONSE TO A RAPIDIY VARYING FIELD; EFFECTS OF "TRAPPED" PARTICLES}

For this case it was desired to choose pext. to represent the charge density on a periodic array of grids,

$$
\rho_{\text {ext. }}(x, t)=\rho \sin \omega_{0} t \sum_{j=-\infty}^{\infty}\{\delta(x-2 j L)-\delta(x-(2 j+I) L)\} \text {, }
$$

and thus to simulate the experiment of Decker and Hirshfield. ${ }^{4}$ The large number of Fourier harmonics required to represent accurately the delta functions led to prohibitively large requirements of computer time, so the somewhat less realistic but computationally more convenient choice of a simple sine wave was made:

$$
\rho_{\text {ext }}(x, t)=2 p_{1} \sin \omega_{0} t \cos k_{0} x,
$$

with $\rho_{1}=$ const. This is just the leading term in the Fourier series expansion of the delta functions for the grids. The initial distribution is taken to be a uniform Maxwellian.

$$
\text { Equations (1) and (2) can be linearized in } p_{\text {ext. }} \text { and }
$$
solved analytically. As a check on the accuracy of the numerical methods (in addition to those previously discussed by Armstrong ${ }^{2,2}$ ), 
Equation (6) was also linearized and integrated numerically for comparison with the long-time limit of the analytical result. For the particular case of $\rho_{1}=0.0458, \mathrm{k}_{0}=0.916$, and $\omega_{0}=1.923$, the two results for $\mathrm{E}_{1}$ agreed to within $0.01 \%$ after the transient part of the solution had Landau-damped away $\left(t \geqslant 10 \omega_{p}^{-1}\right)$.

We are more interested in the non-linear effects on the response to the external field. One class of non-linear phenomena, in particular, can be seen much more easily in the context of the driven problem than in the pure initial value problem: phenomena associated with the oscillations of particles "trapped" in the potential wells of the driving fields.

The potential generated by Equation (7) can be decomposed into right and left traveling waves with phase velocities $\pm \omega_{0} / k_{0}$. A particle moving at approximately the phase velocity of one of the waves sees that wave as essentially a time-independent, constantprofile potential well. (The effect of the non-resonant wave cancels out when viewed on the time-scale of the interaction of the particle with the resonant wave.) If the particle's total energy is small enough it can be considered to be trapped in the potential trough of the wave.

The period of an electron trapped at the bottom of the trough of a wave of amplitude $E_{1}$ and wavenumber $k_{0}$ is $T=2 \pi\left(k_{0} E_{1}\right)^{-\frac{1}{2}}$. 
The period is longer for electrons of greater total energy, and approaches infinity for particles at the boundary of the trapping region. If the wave persists for times longer than the typical oscillation period, we expect to see slow oscillations in the gross properties of the plasma such as total kinetic energy and electrostatic field energy.

Figure 1 shows, for a particular case, the absolute value of the Fourier components of the particle electric field - the total electric field less the external field. The subscripts 1 and 2 indicate components of wavenumber $k_{0}$ and $2 k_{0}$, respectively. The curve labelled P.E. (particle energy) shows the percent change in the total kinetic energy of the particles. In the Fourier-Hermite representation of the distribution, the total kinetic energy in $0 \leq x \leq L$ is given by $(2 \pi)^{\frac{1}{4}}\left(z_{00}+\sqrt{2} z_{20}\right)\left(\pi / 2 k_{0}\right)$. The figure shows that the particle kinetic energy undergoes a slow oscillation with a period of about $32.5{w_{p}}^{-1}$. This value corresponds to the orbital period of particles with a velocity (as measured in the frame of the resonant wave) of about $0.3 \mathrm{v}_{\text {thermal }}$, or about half the width of the trapping region. (These are the particles that occupy the region of greatest distortion of the distribution function, as can be seen in Figure 2.) 
The envelope of the $E_{1}$ curve shows the same slow period superimposed on the oscillations of frequency $\omega_{0} \cdot$ At $t \cong 32$, when the kinetic energy has almost returned to its initial value, $\mathrm{E}_{1}$ undergoes a quick low-amplitude oscillation reminiscent of the phase adjustment it experienced when the external field was switched on. The broad dip of the $E_{1}$ envelope over the time of maximum particle kinetic energy may be due to a detuning of the plasma from the driving frequency $\omega_{0}$ because of the distortion of the distribution function. ( $\omega_{0}$ was chosen to approximately satisfy the dispersion relation for the initial Maxwellian and $\left.\mathrm{k}_{0}=0.916\right)$.

Figure 2 shows the spatially uniform part of the distribution function $f_{0}$ for velocities near $\omega_{o} / k_{0}$ at three times in the energy cycle. The curve for $t=17$ ( $t$ ime of maximum particle kinetic energy) shows the maximum distortion of the distribution from the initial Maxwellian, while the $t=20$ curve shows the decreasing distortion as the trapped particles continue around their orbits in phase space. The trapped particle phenomena are easier to observe in the driven case, where the wave amplitude is maintained, than in the initial value problem where the wave amplitude is decaying by Landau damping. The decaying amplitude may free most of the initially trapped particles before they can complete an appreciable fraction of even one oscillation in the wave trough. This apparently happens in all cases 
shown in Reference 2 except for Figures $2 d$ and 5a, where the first half cycle appears to have been reached by $t \cong 15$.

It is to be emphasized that the distortion of the velocity distribution function near $v \cong \pm \omega_{0} / k$ is of a qualitatively different nature than the development of the quasi-linear "plateau". In the quasi-linear theory ${ }^{6,7,8,9}$ the presence of a continuum of phase velocities provides for a monotonic approach to a flat velocity distribution near the phase velocity of the excited waves; whereas in the case of one driven wave, the distribution first flattens, then develops a hump, then flattens again, and finally returns approximately to its original form.

Because there is a spread in the orbit times for particles in the trapping region, the phenomenon is not entirely reversible. This accounts for the fact that the quantities in Figure 1 do not return exactly to their initial values after the trapped-particle oscillation period. The oscillation in the envelope of $\left|E_{1}\right|$. for example, is presumably subject to a slow damping with an e-folding time which is long compared with the times of any existing computation. Similar considerations have been discussed analytically by Al'tshul and Karpman ${ }^{10}$ and $O^{\prime} \mathrm{Neil}^{I I}$ for the initial value problem. The effect has been observed experimentally by Malmberg and Wharton ${ }^{12}$ for the driven boundary-value problem. 
In time we expect the particles to find themselves evenly distributed around their orbits and the distribution function to have the familiar plateau shape in the vicinity of the phase velocity of the driving wave. However, the time for this to occur is apparently very long compared to the time of any existing computations. 


\title{
4. IANDAU DAMPING OF PERTURBATIONS \\ ON INHHOMOGENEOUS EQUILIBRIA
}

\begin{abstract}
Quite some time ago, Bernstein, Greene, and Kruskal13 and Harris ${ }^{14}$ showed how to analytically construct time-independent solutions to Equations (1) and (2) which involve inhomogeneous electric fields $E(x) \equiv-d \varphi(x) / d x$. Any integrable distribution $f=f\left(\frac{1}{5} v^{2}-\varphi\right)$ which leads to a self-consistent solution for Poisson's equation is such an equilibrium. Armstrong and Montgomery ${ }^{3}$ appear to have found an inhomogeneous equilibrium as the final state of the class of initially unstable electron plasmas they considered. We do not know of previous analytic calculations of inhomogeneous equilibria which would remain numerically stable on the computer. In this section we describe the construction of such equilibria and the study of the damping of perturbations about these equilibria. Analytical studies of increasing degrees of sophistication have been carried out by Montgomery ${ }^{15}$, Low ${ }^{16}$, Pearlstein ${ }^{17}$, Friedberg ${ }^{18}$, and Knorr ${ }^{19}$. For pext. we take a sinusoidal but time-independent value

$$
\rho_{\text {ext. }}=\epsilon \cos \mathrm{k}_{\mathrm{o}} \mathrm{x}
$$


The inhomogeneity is measured by $\epsilon=$ const., and the distribution will be

$$
f=\frac{a}{\sqrt{2 \pi}} \exp \left(-\frac{v^{2}}{2}+\varphi\right) .
$$

We consider solutions for $\varphi$ which can be written as a rapidly converging Fourier series,

$$
\varphi(x)=A_{1} \cos k_{0} x+A_{2} \cos 2 k_{0} x+A_{3} \cos 3 k_{0} x+\ldots,
$$

with $\left|A_{1}\right|>>\left|A_{2}\right|>>\left|A_{3}\right|$, etc., and pick $A_{1}$ sufficiently small that the exponential series $\exp \varphi=1+\varphi+\varphi^{2} / 2 !+\ldots$ also converges rapidly.

Substituting (8) and (9) into (2) and retaining terns through $O\left(\epsilon^{3}\right)$ gives, upon equating total electron and ion charge,

$$
\begin{aligned}
a & =\frac{1}{1+A_{1}^{2} / 4}, \\
A_{2} & =\frac{-a A_{1}^{2} / 4}{a+4 k_{0}^{2}}, \\
A_{3} & =\left(\frac{-a A_{1}}{24}\right)\left(\frac{A_{1}^{2}+12 A_{2}}{a+9 k_{0}^{2}}\right), \\
\epsilon & =2 \rho_{1}=A_{1}\left(a+k_{0}^{2}\right)+\frac{a A_{1}}{8}\left(A_{1}^{2}+4 A_{2}\right) .
\end{aligned}
$$


It is most convenient to choose $A_{1}$ and $k_{0}$ and calculate the rest of the quantities using relations (10). From these the equilibrium coefficients $Z_{m n}$ are easily calculated, since the velocity distribution is just Maxwellian. Coefficients with $m \neq 0$ vanish identically. The computed $z_{\text {on }}$ are then used as initial conditions to see how well they represent a time-independent solution of Equation (6). For $A_{I}=0.1$ and $k_{0} \leq 0.5$, the $z_{m n}$ matrix was stationary through three significant figures with oscillations of only \pm . in the fourth figure. (The I.B.M. 7044 on which these computations were done does 8-digit arithmetic.) We found that adjustments of less than $0.05 \%$ in the initial matrix elements improved the degree to which the solution was stationary to the extent of moving the oscillations out to the sixth or seventh significant figure. This "tuning" is desirable to get as near as possible to a stationary solution so that small perturbations from the solution can be studied for longer times. For $\mathrm{k}_{\mathrm{o}}=0.75$ the calculated equilibrium was off by about one per cent, but was easily corrected with a few trials.

Once an equilibrium set of $Z_{00}, z_{01}$, and $z_{02}$ is established for a given $\mathrm{k}_{\mathrm{o}}$, inhomogeneous Landau-damping for that wavenumber is measured by using an initial value of $\mathrm{z}_{\mathrm{ol}}$ (perturbed) $\cong 1.05 \mathrm{Z}_{\mathrm{ol}}$ (equilibrium), and watching the corresponding electric field perturbation, $E_{1}$ pert.' damp for several cycles. A sample measurement for 
the case of $k_{0}=0.5$ and $A_{1}=0.1$ is shown in Figure 3. The slopes of the two straight lines represent reasonable error limits for this measurement of the damping decrement $Y$. The unperturbed equilibrium value of $z_{02}$ was used for this run but a perturbation electric field of wavenumber $2 \mathrm{k}_{\mathrm{o}}$ was soon generated nonlinearly. This perturbation field, $\mathrm{F}_{2}$ pert.' stayed at least a factor of 20 smaller than $\mathrm{E}_{1}$ pert. so the damping can be considered to be essentially linear.

$$
\text { Damping decrements measured in this way for } A_{1}=0.1 \text { and }
$$

for several values of $k_{0}$ are shown in Figure 4. Error bars indicate uncertainties in the values when uncertainties are larger than the plotted points. Solid circles represent cases where the perturbation had the same wavenumber as the inhomogeneity; open circles represent cases where the perturbation wavenumber was twice the inhomogeneity wavenumber. In the latter cases the equilibrium values of $z_{o O}$ and $Z_{01}$ were used but $Z_{02}$ was started $10 \%$ larger than its equilibrium value. For perturbations of wavenumber $2 \mathrm{k}_{0}=0.50$ and 0.75 the measured damping rates fell within the error limits of rates measured for $k_{0}=0.50$ and 0.75 and are not shown in Figure 4. The solid curve shows $Y\left(k_{0}\right)$ calculated ${ }^{20}$ for linear Landau damping about homogeneous equilibria $\left(A_{1}=A_{2}=A_{3}=0\right)$.

As $k_{0}$ decreases the measured values of $V\left(k_{0}\right)$ lie farther above the homogeneous damping curve. The small $\mathrm{k}_{0}$ limit is the domain 
of applicability of the inhomogeneous Landau damping calculations of Jackson and Raether ${ }^{21}$. Their calculations indicated that for small ko Landau damping is greatly increased by even small background inhomogeneities.

The curve in Figure 5 shows the increase in the damping decrement $\gamma$ with increasing background inhomogeneity $\rho_{1}(=v / 2$ in Jackson and Raether21) where the eigenfunction of the electric field is assumed to be the Mathieu function $\operatorname{se}_{2}\left(k_{0} x, q\right)$ with $q=4 \rho_{1} /\left(3 k_{0}^{2}\right)$. The condition for $\mathrm{se}_{2}$ to be the eigenfunction is that $a=\left(\omega^{2}-1\right) /\left(3 k_{0}^{2}\right) \approx 4$ or $\omega^{2}=1+12 k_{0}^{2}$ for $q \leqslant 1.5$. The points in Figure 5 show damping rates obtained by least-squares fits to data similar to that presented in Figure 3. In the present case damping is very much slower and the perturbation field is calculated for as long as $45 \mathrm{w}_{\mathrm{p}}^{-1}$. The error bars are drawn conservatively and represent the difficulty in measuring very slow damping of small perturbations. The condition for $\mathrm{se}_{2}$ is not accurately satisfied in these calculations - $\omega$ is found to be about 1.10 instead of 1.25. Nevertheless, the agreement in the damping decrements is striking. 


\section{RESPONSE TO A SLOWLY VARYING FIELD $\left(\omega<<\omega_{\mathrm{p}}\right)$; ISOTHERMAL BEHAVIOR OF AN ELECTRON PLASMA}

Many plasma phenomena involving ions are characterized by time scales slow compared to the electron plasma frequency. This permits the more mobile electrons to follow the ion motion while remaining in a quasi-steady state configuration. Ion acoustic waves and drift waves are well-known examples. In many situationsaz,a3 it greatly simplifies the mathematics to assume that the electron gas obeys a locally isothermal equation of state. In our onedimensional situation, this means that the ratio $P(x, t) / P_{m}(x, t)$ is constant, where $\mathrm{P}$ is the electron pressure computed from the distribution function and $\rho_{m}$ is the local electron mass density. In this section we check the constancy of the ratio over position and time while we slowly vary the background ion inhomogeneity. Again we use $\rho_{\text {ext. }}=2_{\rho_{1}}$ sin $\omega_{0} t \cos k_{0} x_{\text {. }}$ The wavenumber used for these tests was $k_{0}=0.75$ which corresponds to electron oscillations of frequency $\omega(\mathrm{k})=1.73$. The ion frequency was chosen much smaller: $\omega_{0}=0.75$. The integrations were carried out for $41.9 \omega_{p}^{-1}$ (one-half of an ion cycle) for two kinds of initial conditions. In one case the ion perturbation was started at its maximum value $\left(\rho_{\text {ext. }} \propto \cos \omega_{\mathrm{o}} t\right)$ and the electrons were in the inhomogeneous equilibrium 
appropriate to $\mathrm{k}_{0}$ and $\rho_{1}$ and in two other cases the ion perturbation was started from zero $\left(\rho_{\text {ext. }} \propto \sin \omega_{0} t\right)$ and the electrons were spatially uniform. To reduce computing time for these tests we employed the Brownian - motion type of Fokker - Planck collision term previously used by Lenard and Bernstein 24, Grant and Feix ${ }^{25}$, and Armstrong and Montgomery ${ }^{3}$ :

$$
\left(\frac{\partial f}{\partial x}\right)_{c}=v_{c}\left(\frac{\partial}{\partial v}(v f)+\frac{\partial^{2} f}{\partial v^{2}}\right) \text {. }
$$

In the Fourier-Hermite representation the term is merely - $\nu_{c} \mathrm{~m} z_{m n}$ on the right hand side of Equation (6). Since the term is proportional to $m$ it has only a small effect on the low order terms in the Hermite expansion. Even for the small "collision frequency" $\nu_{c}=0.002$ that was used in these tests the effect on terms with larger $m$ is sufficient to smooth out the short-wavelength velocity-space wrinkles in the distribution function and to limit expansion of the matrix in the Hermite direction. For additional discussion of the collision term see references 3 and 25 .

The left hand panel of Figure 6 illustrates the effect of the collision term for the case with $\rho_{\text {ext }} \propto \cos \omega_{0} t$. The second order departure $f_{0}^{(2)}(v, t)$ of the spatially uniform part $f_{0}(v, t)$ 
of the distribution function from its initial Maxwellian $f_{0}(v, t=0)$ is shown for $t=$ one-quarter ion cycle. One curve resulted from $\nu_{c}=0.002$ and the other from $\nu_{c}=0$. The collision term eliminated the velocity-space wrinkles and reduced the departure from the Maxwellian. The right hand panel shows $f_{0}^{(2)}$ calculated after onehalf ion cycle using $v_{c}=0.002$.

Variations in the ratio $\mathrm{P} / \mathrm{P}_{\mathrm{m}}$ are shown in Figure 7 for three cases. Panel (a) shows the ratio as a function of time at $x=0$ (the "worst" choice of $\mathrm{x}$, from the point of view of the constancy of $\left.\mathrm{P} / \mathrm{O}_{\mathrm{m}}\right)$, and panel (b) shows the ratio as a function of $x$ at the time of maximum ion perturbation (again the "worst" choice). The figure shows that the ratio was preserved to about $2 \%$ when the total perturbation was $2 \rho_{1}=0.156(\cong 15 \%)$, and to about $7 \%$ when the total perturbation was $2_{\rho_{1}}=0.312$.

Although the calculations were carried out for only $41.9 \omega_{p}^{-1}$, we feel that these results furnish good support for the assumption that electrons obey a locally isothermal equation of state in the presence of a slowly varying ion background density.

We remark that even thougn the locally isothermal behavior of the electron distribution does seem to be well borne out by the computation, the assumptions made ${ }^{23}$ on the form of the electron distribution function in order to deduce the loca? Iy isothermal 
relation do not themselves seem to be particularly well borne out. An analytical demonstration of the locally isothermal law has apparentIy not been given for the case of a slowly varying external potential. 


\section{APPROACH TO INHOMOGENEOUS EQUILIBRIA; DEVELOPMENT OF A SHIELDING CLOUD}

In Section 4 we measured damping of small perturbations about an inhonogeneous equilibrium and in section 5 we isothermally led a uniform plasma to an inhomogeneous equilibriun by slowly varying the background charge density. In this section we consider the approach to an inhomogeneous equilibrium when the background inhomoreneity is $\underline{\text { suddenly }}$ switched on $\left(\rho_{\text {ext. }}=0, t<0 ; \rho_{\text {ext. }}=2 \rho_{1} \cos k_{0} x, t \geq 0\right)$. The electrons rush to screen out the external field. They initially overcompensate for the inhomogeneity and then undergo Landau damped oscillations as they approach a new equilibrium. If $\rho_{1}$ is not too large, then the spatial charge distribution is the same as for the equilibrium calculated in Section 4. The velocity dependence, however, will not be Maxwellian in this case and $f_{1}$ and $f_{2}$ will be marked by large velocity-space wrinkles. The solution of the linearized Vlasov equation for this case gives the total electric field in the long-time limit $E_{1 \text { TOT }}=E_{\text {ext. }} / D\left(\mathrm{k}_{0}, 0\right)$ where $E_{\text {ext. }}=-i 0_{1} / \mathrm{k}_{0}$ and $\mathrm{D}\left(\mathrm{k}_{0}, \mathrm{O}\right)$ is the plasma dielectric function for zero frequency. Figure 8 shows an example of the damping of $\mathrm{E}_{1}$ TOT for $k_{0}=0.50$ and $\rho_{1}=0.05$. The envelope curves are for a constant damping rate fitted to the first three extrema of the $\mathrm{E}_{1} \mathrm{TOT}$ curve. 
Even for this small external field the nonlinear effect.s are sufficient to shift the equilibrium value of $E_{1}$ TOT away from the linear prediction. The external field can be thought of as a wave with zero phase veiocity, and, as we see in Figure 9, the spatially uniform part of the particle distribution is most strongly affected in the trapping region surrounding $\mathrm{v}=0$.

If $\rho_{1}$ were made much larger, then the nonlinear terms would predominate, and a plot such as Figure 8 would show no sign that $\mathrm{E}_{\text {I TOT }}$ approaches an equilibrium in the time intervals that can be calculated. 


\section{ACKNOWLEDGEMENTS}

The author is indebted to Professor David Montgomery for suggesting this investigation and for his guidance throughout its course. At the outset the numerical work was expedited greatly by suggestions and sample computer codes generously provided by Dr. Thomas P. Armstrong. It is also a pleasure to acknowledge fruitful discussions with Dr. Georg Knorr and Dr. S. Peter Gary. The work was supported in part by the National Aeronautics and Space Administration under Grant No. NGR-16-001-043. 
IArmstrong, T. P., Ph.D. Thesis, University of Iowa Report 66-34 $(1966)$.

2Armstrong, T. P., Phys. Fluids 10, 1269 (1967).

3Armstrong, T. P., and D. Montgomery, J. Plasma Physics 1.425 (1967).

4 Decker, J. F., and J. L. Hirshfield, Proc. Conf. on Quiescent Plasmas (Frascati 1967), Vol. II, p. 475.

5Gill, S., Proc. Cambridge Phil. Soc. 47, 96 (1951).

GVedenov, A., E. Velikhov, and R. Sagdeev, Nucl. Fusion Suppl. Pt. 2, 465 (1962).

"Drummond, W. E., and D. Pines, Nucl. Fusion Suppl. Pt. 3, 1049 (1962).

8Frieman, E., and P. Rutherford, Ann. Phys. (N. Y.) 28, 134 (1964).

${ }^{9}$ Bernstein, I. B., and F. Engelmann, Phys. Fluids 2, 937 (1966).

${ }^{\circ 0} \mathrm{Al}$ 'tshul', L. M., and V. I. Karpman, Zh. Eksperim. i Teor. Fiz. 49, 515 (1965). English transl.: Soviet Phys.-JETP 22, 361 (1966).

IIO'Neil, T., Phys. Fluids 8, 2255 (1965).

I2Malmberg, J. H., and C. B. Wharton, Phys. Rev. Lett. 19, 775 (1967).

I3Bernstein, I. B., J. M. Greene, and M. D. Kruskal. Phys. Rev. 108. 546 (1957).

I4Harris, E. G., Bull. Amer - Phys. Soc. Ser. II, 2․ 67 (1957).

I5Montgomery, D., Phys. Fluids 3, 274 (1960).

${ }^{16}$ Low, F. E., Phys. Fluids 4, 842 (1961).

I"Pearlstein, L. D., Phys. Fluids 7, 1461 (1964). 
$1 \&$ Freidberg, J. P., Phys. Fluids 8, 1031 (1965).

19 Knorr, G., U. of Iowa Report 67-65 (1967) (to be published in Phys. Fluids).

zo Jackson, J. D., J. Nucl. Energy, Part C: Plasma Phys. 1, 171 (1960). The numerical values for this curve were kindly supplied by S. Peter Gary, U. of Iowa.

21 Jackson, E. A., and M. Raether, Phys. Fluids 2, 1257 (1966). 22Tasso, H., Phys. Letters 24A, 618 (1967).

23Montgomery, D., Phys. Rev. Letters 19, 1465 (1967).

24 Lenard, A., and I. B. Bernstein, Phys. Rev. 112, 1456 (1958).

25Grant, F. C., and M. R. Feix, Phys. Fluids 10, 696 (1967). 


\section{FIGURE CAPTIONS}

FIGURE 1. Aosolute value of first and second Fourier components of particle electric field for an electron plasma driven by a sine wave. Curve labelled P.E. shows the percent increase in the total electron kinetic energy. Total electric field is particle field plus the external field.

FIGURE 2. Distortion of the spatially uniform part of the distribution function near the phase velocity $\omega_{0} / k_{0}$ of the driving wave for the situation of Figure 1. The distortion is greatest at $T \approx 17 \omega_{p}^{-1}$, approximately one-half of the period of trapped particle motion.

FIGURE 3. Damping of perturbation on a spatially non-uniform equilibrium to illustrate measurement of the damping decrement $\gamma$. Difference in slopes of the two straight lines indicates the uncertainty for this measurement.

FIGURE 4. Landau damping decrement as a function of wavenumber for perturbations on spatially non-uniform equilibria. The solid curve is the corresponding spatially uniform rate. Error bars indicate uncertainties in the measurement of the damping decrement.

FIGURE 5. Landau damping decrement as a function of inhomogeneity $p_{1}$ for $k_{0}=0.22$. The theoretical curve (based on Jackson and Raether) assumes that the eigenfunction of the electric field is the Mathieu function $\mathrm{se}_{2}\left(\mathrm{k}_{0} \mathrm{x}, \mathrm{q}\right)$. 
FIGURE 6. Second order change $f_{0}^{(2)}$ in the spatially uniform part of the distribution function from its initial Maxwellian after one-quarter ion cycle (left panel) and after one-half ion cycle (right panel). The left panel also illustrates the effect of leaving the collision term out of the calculation. $f_{0}^{(2)}$ is measured in percent of $f_{0}(v=0, t=0)$.

FIGURE 7. (a) Percent variation in the ratio of electron pressure to mass density as a function of time at $x=0$ for three cases. Zero variation represents perfect isothermal response of electrons to the slow variation in the ion background density.

(b) Same as (a) except variation is now shown as a function of $x$ at the time of maximum ion inhomogeneity for each case.

FIGURE 8. Damping of the first Fourier component of the total electric field as electrons approach an inhomogeneous equilibrium to screen out a fixed external charge density ( $\left.o_{\text {ext. }}=2 p_{1} \cos k_{0} x\right)$. The envelope curves represent purely expontential damping fitted to the first three extrema of $\mathrm{E}_{1} \mathrm{TOT}$. Nonlinear effects shift the equilibrium value from the linear prediction.

FIGURE 9. Modification of the spatially uniform part of the electron distribution in the vicinity of $\mathrm{v}=0$ for the situation of Figure 8. 


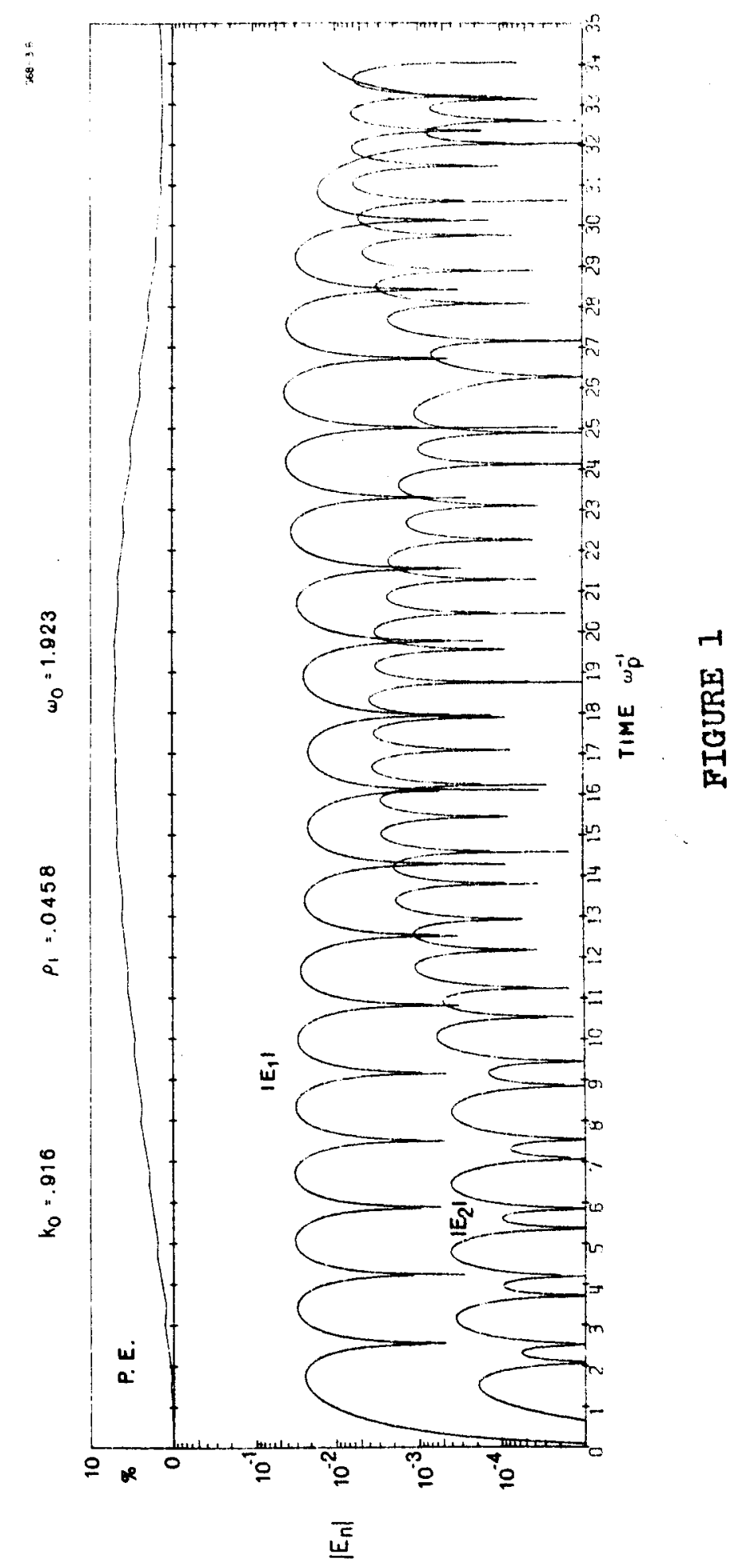


G68-228-1

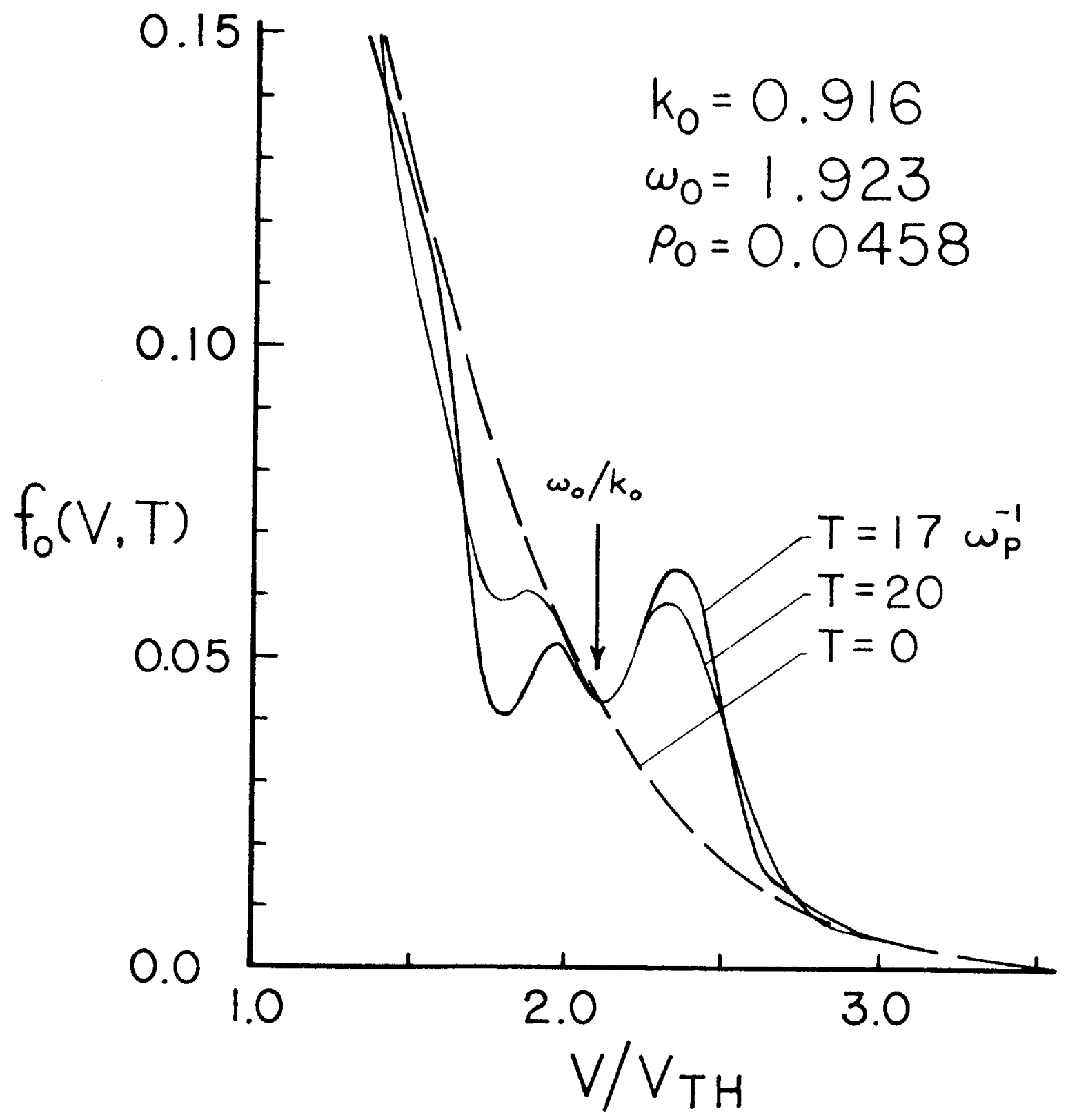

FIGURE 2 


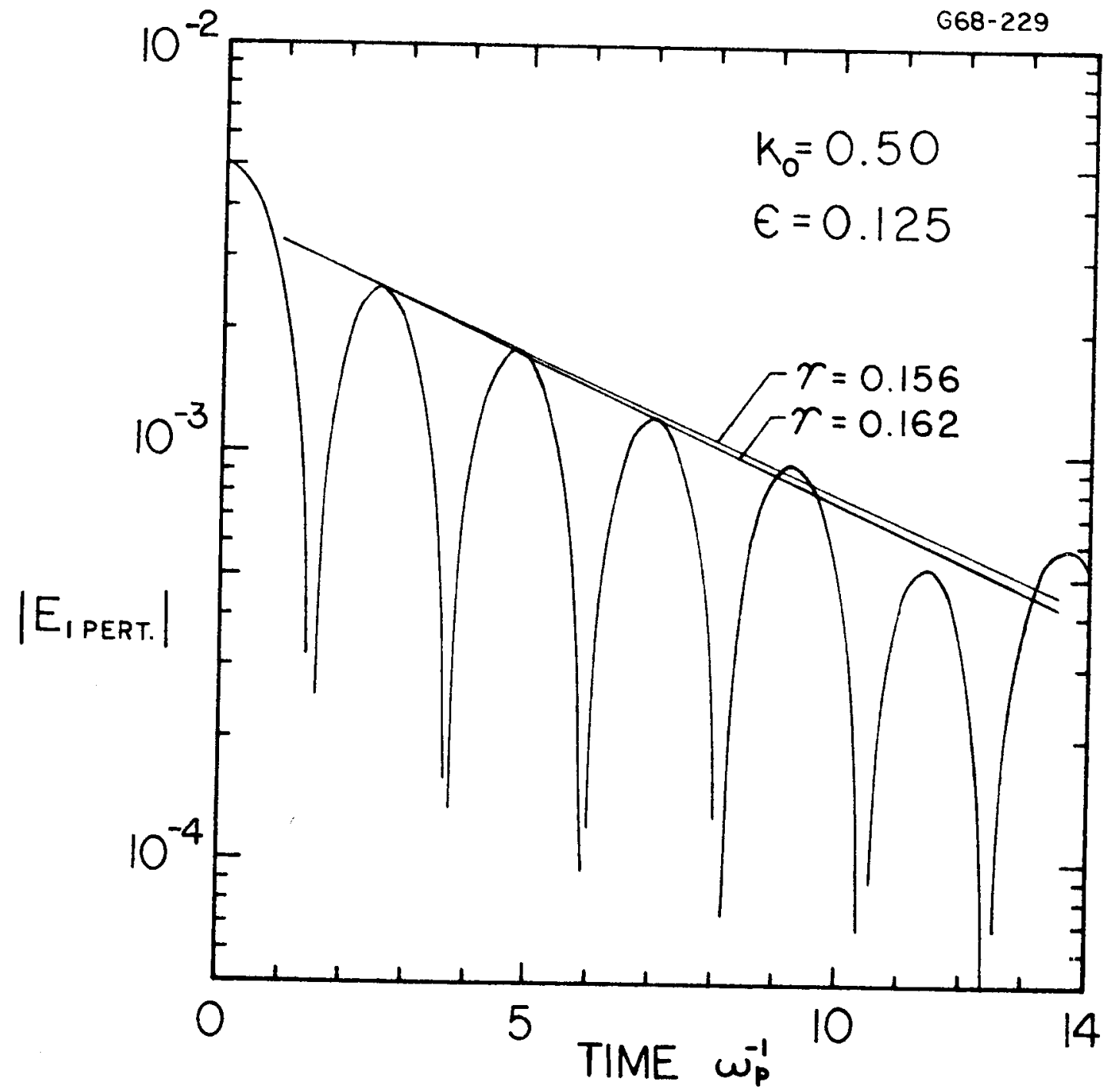

FIGURE 3 


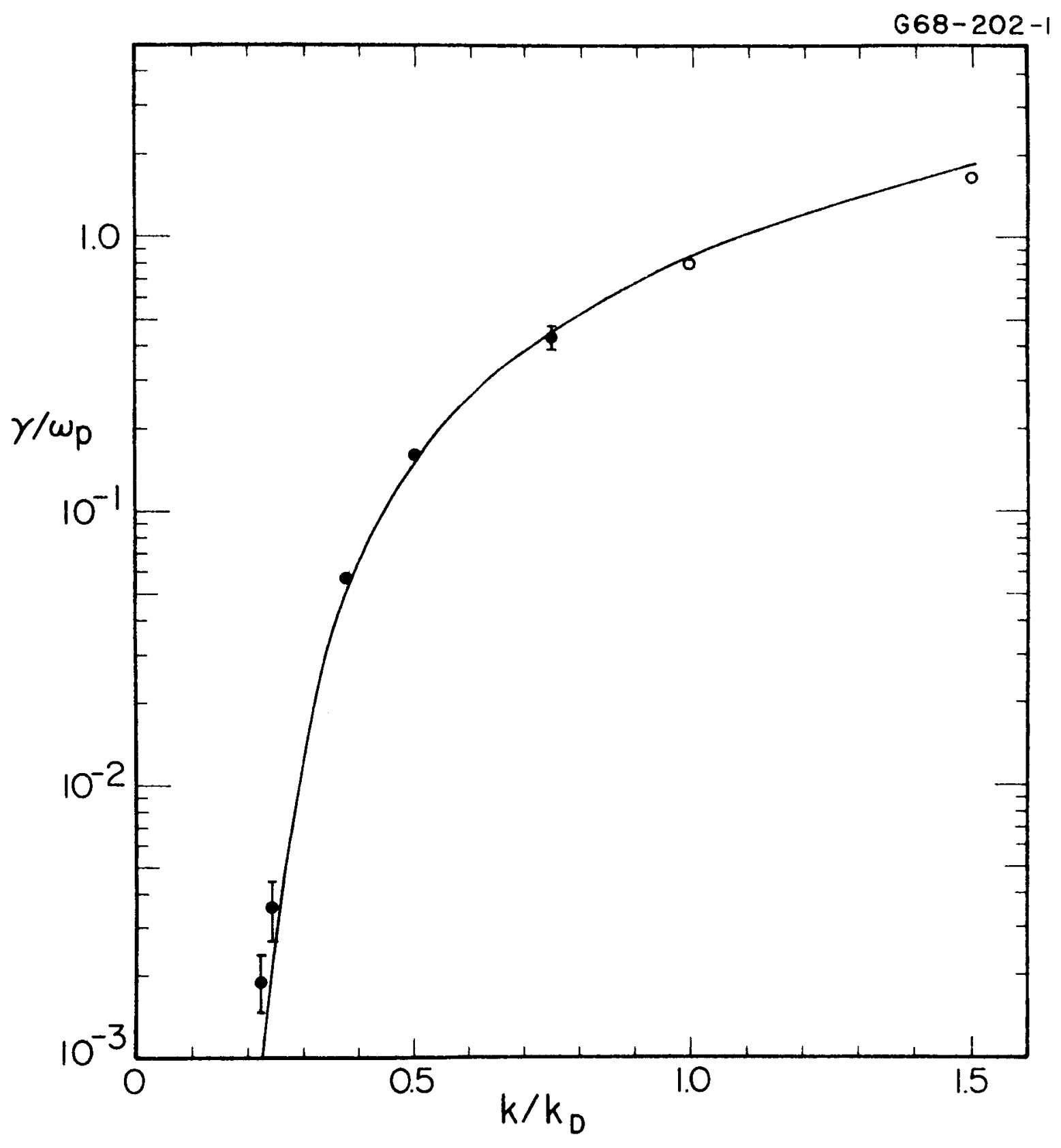

FIGURE 4 


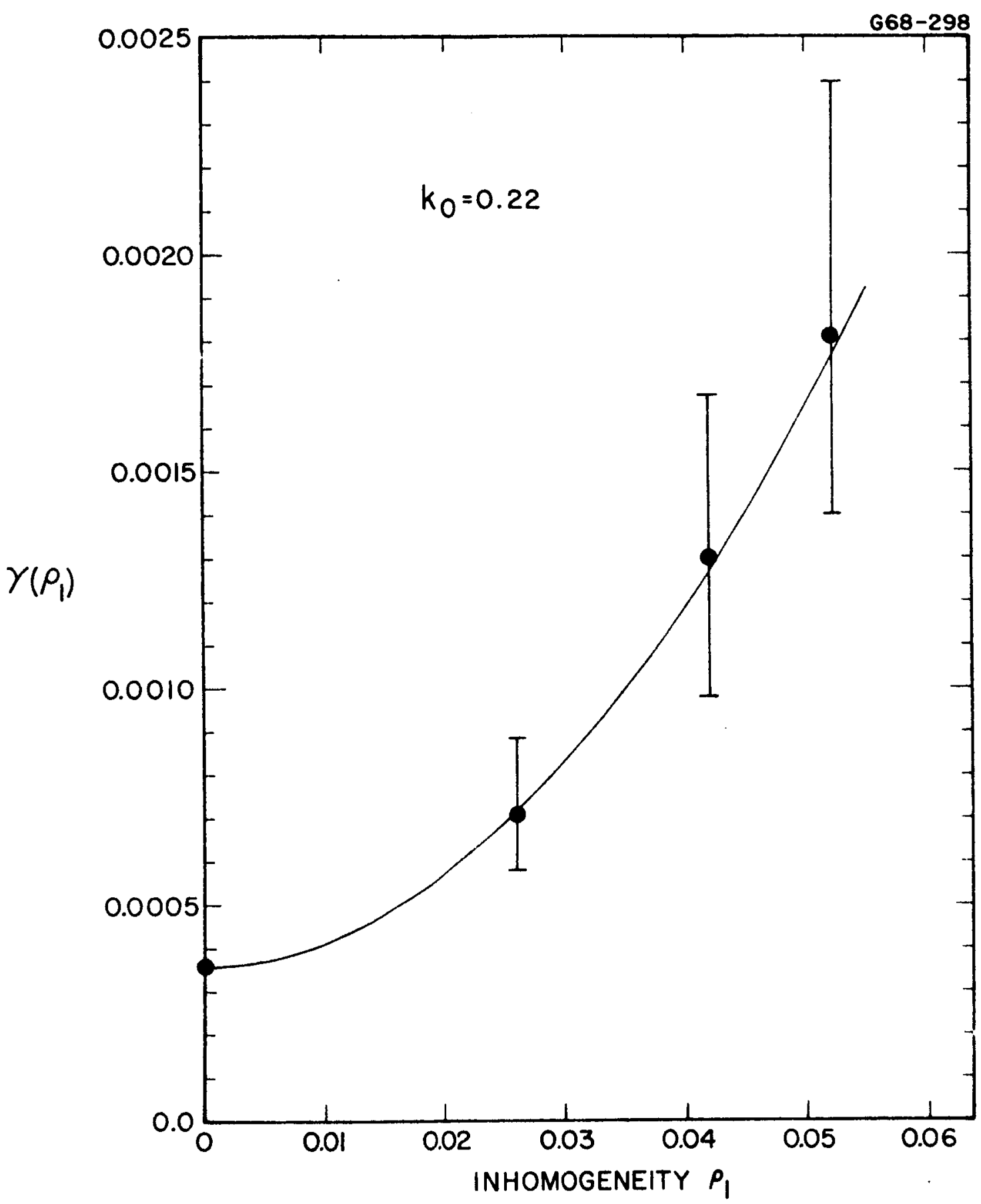

FIGURE 5 


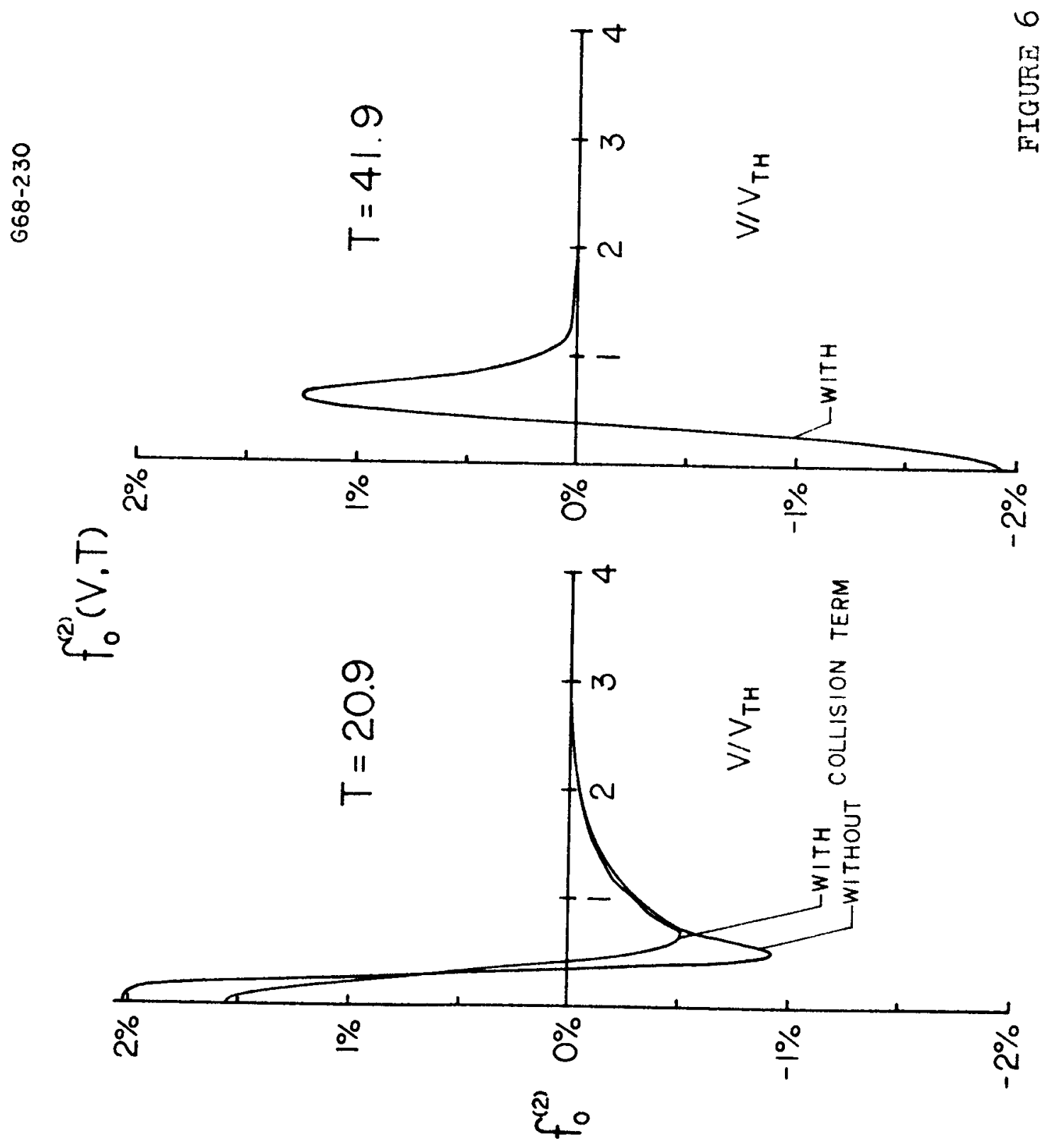




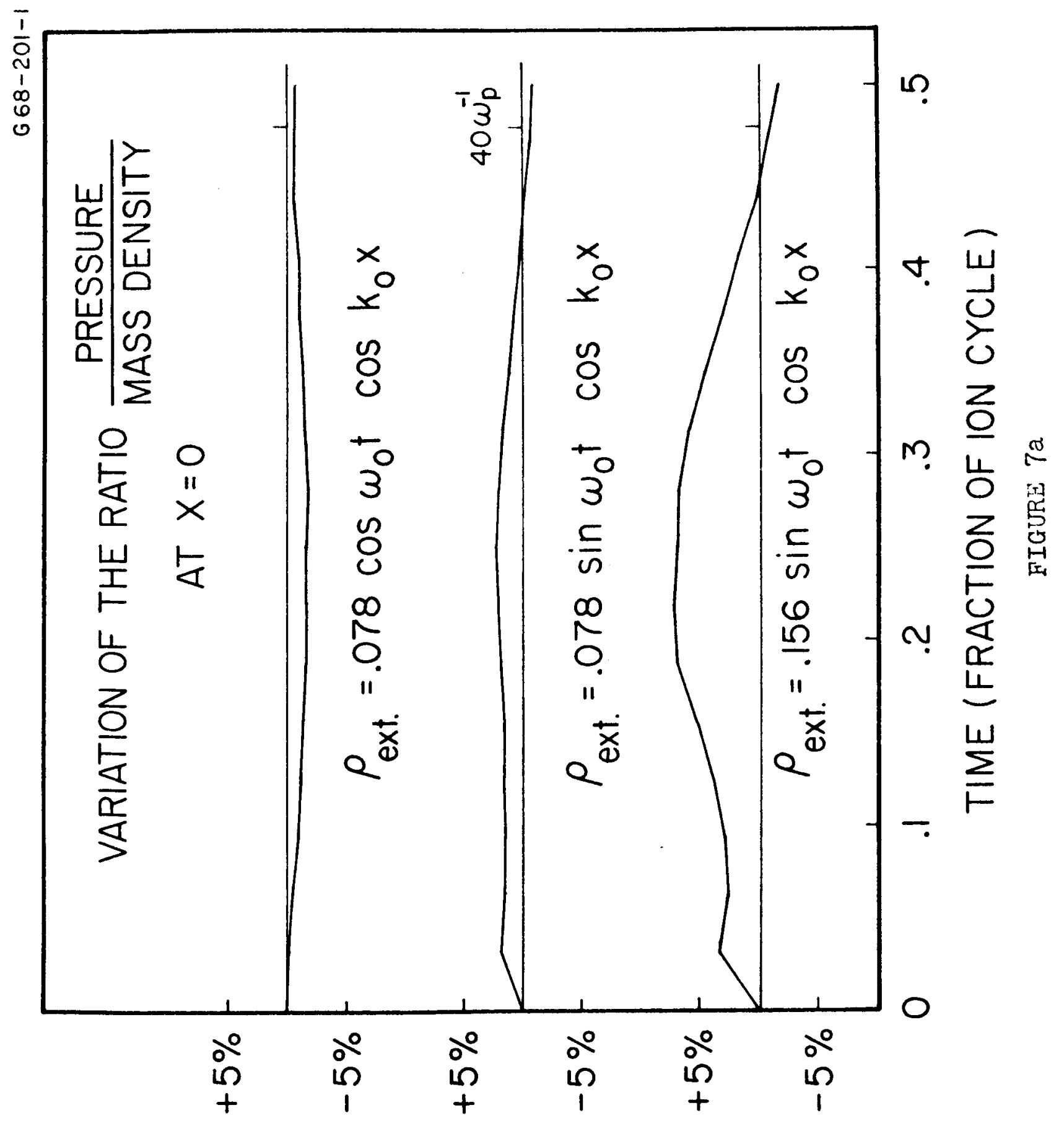




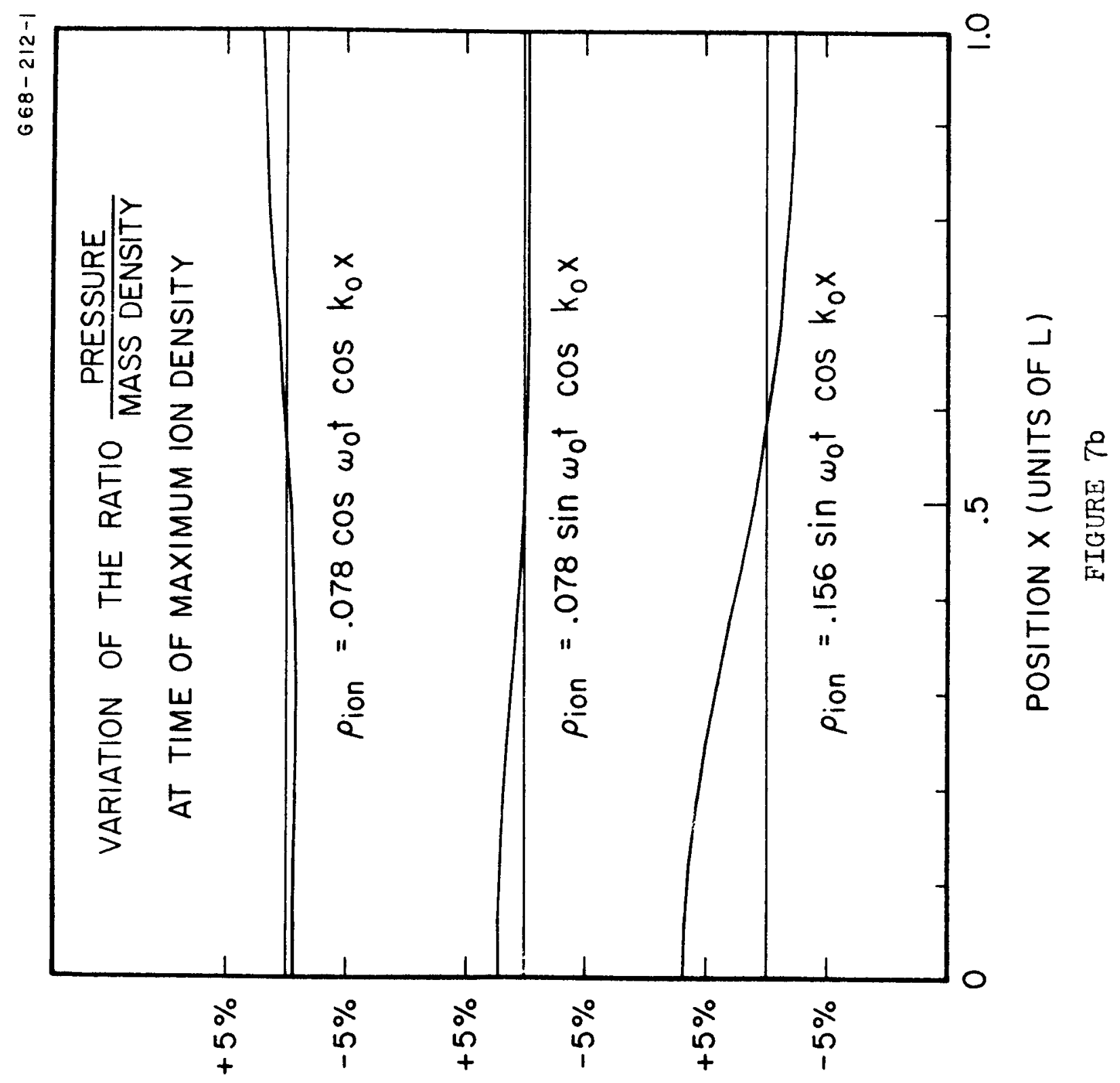




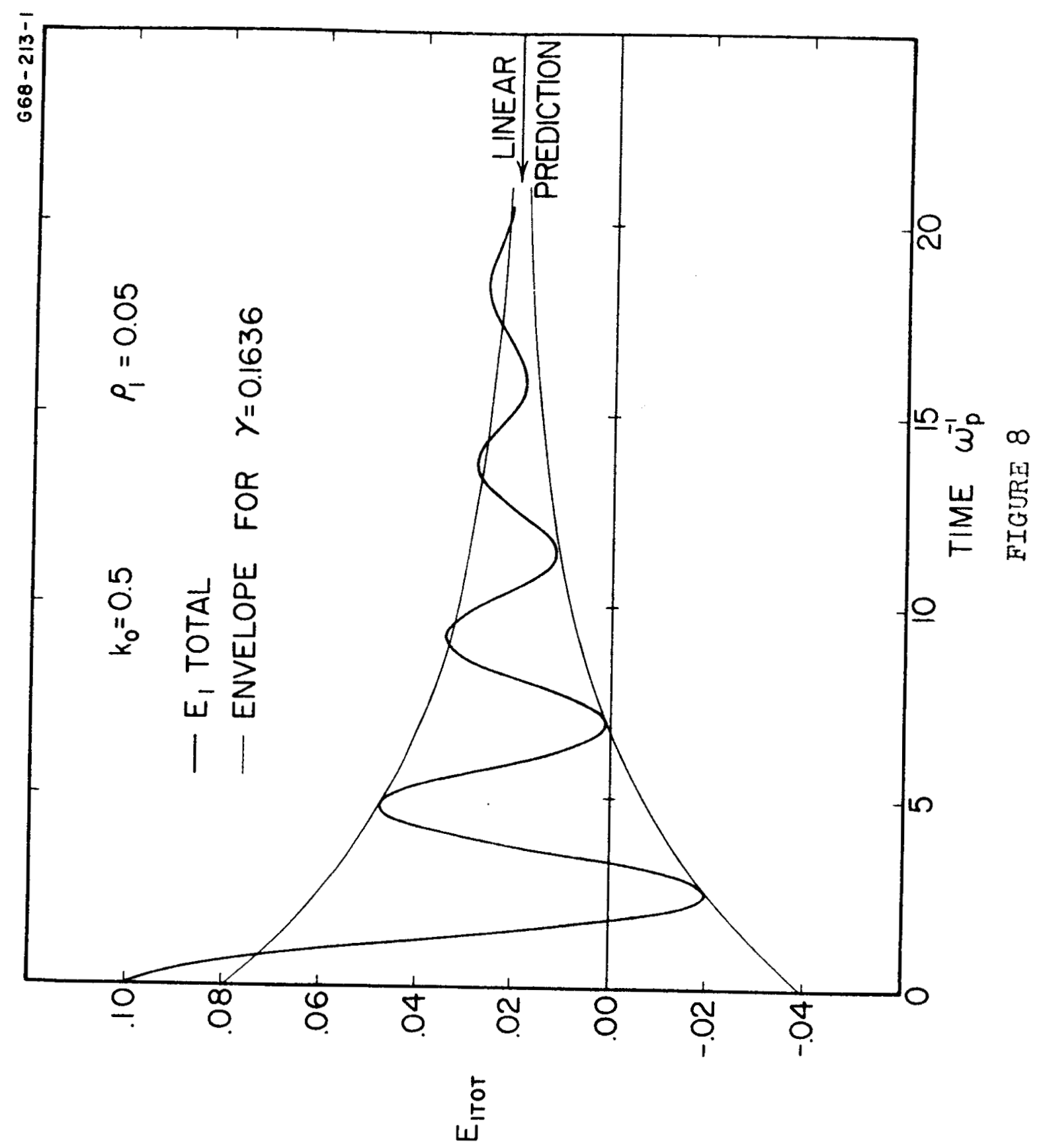




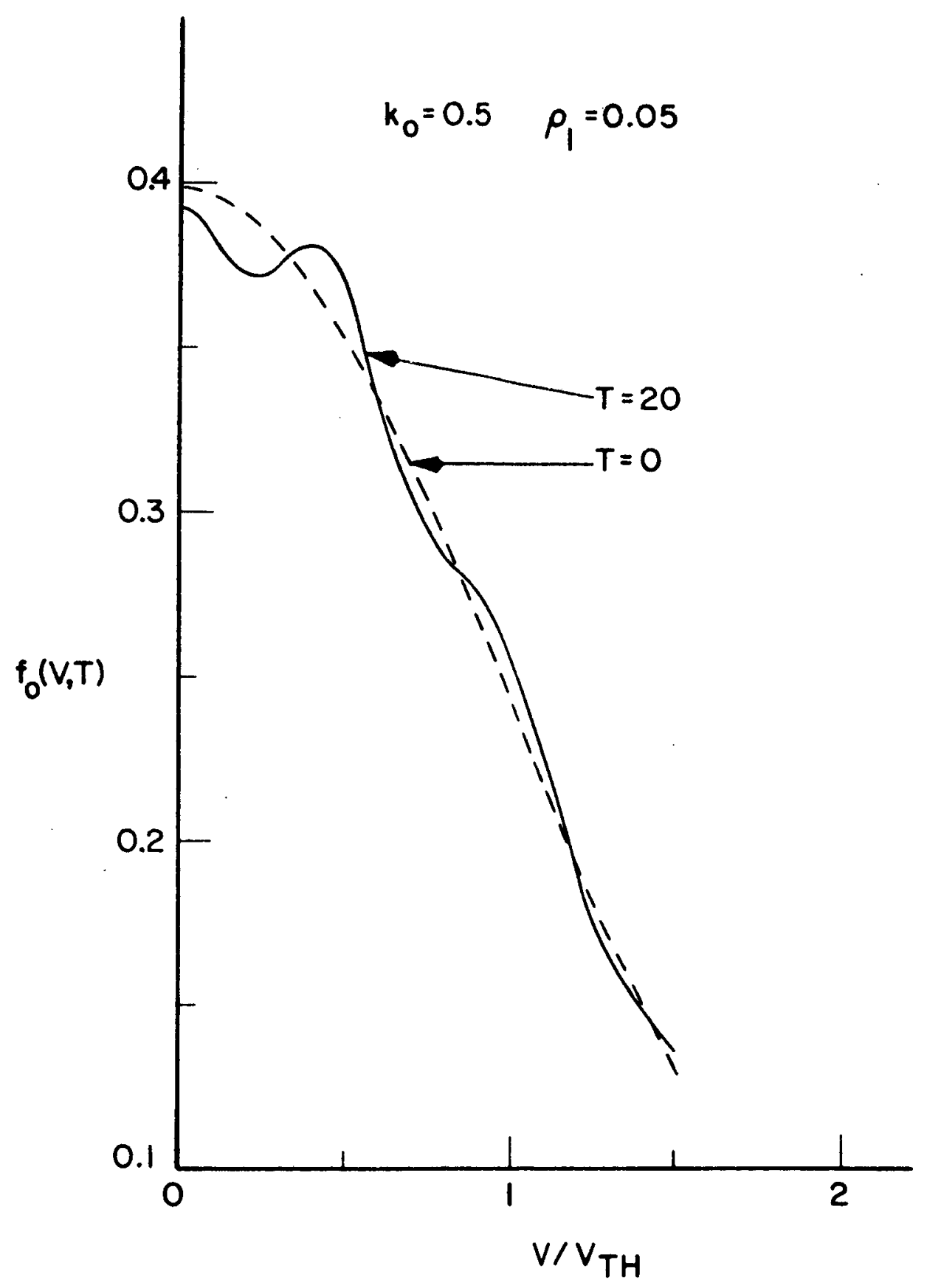

G68-26I

FIGURE 9 
"Response of a One-Dimensional Vlasov Plasma to External Electric Fields," by Rollin C. Harding (U. of Iowa 68-15)

(I) Page 21, 4th line from the bottom:

$$
\text { Replace } \omega_{0}=0.75 \text { by } \omega_{0}=0.075
$$

(2) Page 22, only equation on page:

Replace $\quad\left(\frac{\partial f}{\partial x}\right)_{c}$ by $\quad\left(\frac{\partial f}{\partial t}\right)_{c}$.

